Pacific Journal of Mathematics

FATOU'S LEMMA IN NORMED LINEAR SPACES 


\section{FATOU'S LEMMA IN NORMED LINEAR SPACES}

\section{STEPHEN SChEINBERG}

This note presents a generalization of Fatou's lemma to arbitrary normed linear spaces. Several examples illustrate the situations in which this notion is meaningful. The main theorem gives an abstract characterization of the Fatou property. In particular this resolves the case of any reflexive space. An example shows that Fatou's lemma may fail even for uniform convergence in a normed algebra of continuous functions.

Frequently in analysis one obtains a function by a limiting process which is weaker (less demanding) than convergence in the norm. For example, a continuous function may be obtained as the point-wise, but not necessary uniform, limit of other continuous functions. Even though the limit is not a norm limit, one may still need to know that the norm of the limit function is no greater than the norms of the approximating functions. The classical case is, of course, Fatou's lemma: if $f_{n} \rightarrow f$ pointwise, then

$$
\int|f| \leqq \lim \text { inf } \int\left|f_{n}\right| \text {. }
$$

Another common situation is this. A subspace $A \subseteq C(X)$ is given which has a norm, $\|f\| \geqq \sup |f|$. If $f_{n} \rightarrow f$ pointwise (or uniformly), does it follow that $\|f\| \leqq \lim$ inf $\left\|f_{n}\right\|$ ? The answer is "yes" quite often, but can be "no," even when $A$ is a subalgebra.

Motivated by a wide variety of examples, I wish to consider the following general situation. Throughout this paper $A$ will be a normed linear space, not necessarily complete, and $\mathscr{T}$ will be a locally convex Hausdorff topology on $A$ which is weaker (coarser) than the norm topology. Say that Fatou's lemma holds for $A$ relative to $\mathscr{T}$ if whenever $a_{\beta} \rightarrow a$ in $\mathscr{T}$, it follows that $\|a\| \leqq \lim _{\gamma} \inf _{\beta \geqq r}\left\|a_{\beta}\right\|$. It is usually easier to apply the equivalent condition stated in the following proposition.

Proposition. Fatou's lemma holds for $A$ relative to $\mathscr{T} \Leftrightarrow$ whenever $a_{\beta} \rightarrow a$ in $\mathscr{T}$, it follows that $\|a\| \leqq \sup _{\beta}\left\|a_{\beta}\right\|$.

Proof. $\Rightarrow$ is obvious, since sup $\geqq \lim$ inf. For $\Longleftarrow$, let $a_{\beta} \rightarrow a$ in $\mathscr{T}$ be given. We may assume $\lim _{r} \inf _{\beta \geq \gamma}\left\|a_{\beta}\right\|=L<\infty$, for otherwise there is nothing to prove. Fix $\varepsilon>0$. Given $\gamma, \exists \beta \geqq \gamma$ with $\left\|a_{\beta}\right\| \leqq L+\varepsilon$. Write this $\beta$ as $\beta(\gamma)$ and we obtain $a_{\beta(\gamma)} \rightarrow a$ with $\sup _{r}\left\|a_{\beta(r)}\right\| \leqq L+\varepsilon$. By hypothesis, then $\|a\| \leqq L+\varepsilon$. Now let $\varepsilon \rightarrow 0$. 
It may be instructive to examine a few examples.

1. For each $E_{1} \subseteq E_{2} \subseteq E_{3} \subseteq \cdots$ with each $E_{j}$ measurable and $\cup E_{j}=[0,1]$, the topology $\mathscr{T}$ of uniform convergence on every $E_{j}$ is locally convex and Hausdorff. Egoroff's theorem shows that if measurable $f_{n} \rightarrow f$ pointwise, then $f_{n} \rightarrow f$ in $\mathscr{T}$ for some such $\mathscr{T}$. In this manner the classical Fatou's lemma becomes a special case of the definition above.

2. Let $A=C^{1}[0,1],\|f\|=\sup |f|+\sup \left|f^{\prime}\right|$, and $\mathscr{T}$ be the topology of pointwise convergence. To verify Fatou's lemma, suppose $f_{\alpha}(x) \rightarrow f(x)$ for each $x$. Let $x_{0}$ maximize $|f|$ and $x_{1}$ maximize $\left|f^{\prime}\right|$. Then $\left[f_{\alpha}(x+\delta)-f_{\alpha}(x)\right] / \delta \underset{\alpha}{\rightarrow}[f(x+\delta)-f(x)] / \delta$, implying $f^{\prime}{ }_{\alpha}\left(x_{1}+\theta_{\alpha} \delta\right) \underset{\alpha}{\longrightarrow}$ $f^{\prime}\left(x_{1}+\theta \delta\right)$ by the mean-value theorem. This takes care of the part of the norm depending $\left|f^{\prime}\right|$, the part depending on $|f|$ following from $f_{\alpha}\left(x_{0}\right) \underset{\alpha}{\rightarrow} f\left(x_{0}\right)$. The same result holds for $\|f\|=\sup \left(|f|+\left|f^{\prime}\right|\right)$ or $\|f\|=\max \left(\sup |f|\right.$, sup $\left.\left|f^{\prime}\right|\right)$, etc. Using the appropriate differential quotients we can extend this example to any of the usual $C^{k}$ norms and of course to other manifolds.

3. Let $D$ be an open connected subset of the plane which is the interior of its closure $\bar{D}$. Let $A=$ all functions continuous on $\bar{D}$ which are analytic on $D$, with the sup norm, and $\mathscr{T}=$ the topology of convergence in each derivative at some fixed point $z_{0} \in D$. To conclude $\|f\| \leqq \sup \left\|f_{\alpha}\right\|$ from $f_{\alpha}^{(k)}\left(z_{0}\right) \rightarrow f^{(k)}\left(z_{0}\right)$ (all $k$ ), one may argue this way: if sup $\left\|f_{\alpha}\right\|=\infty$, done; if $\sup \left\|f_{\alpha}\right\|<\infty$, then a standard normal families argument shows that some subnet converges uniformly on compact subsets of $D$. This implies that

$$
|f(z)| \leqq \sup _{\alpha}\left|f_{\alpha}(z)\right| \leqq \sup _{\alpha}\left\|f_{\alpha}\right\|
$$

for each $z \in D$, which completes the proof. See de Leeuw [1] for a different proof of a similar example.

4. Let $A=$ all continuous functions on $[0,1]$ satisfying $\|f\|=$ $\sup |f|+\Sigma 2^{n}|f(1 / n)|<\infty$ and let $\mathscr{T}$ be the topology of pointwise convergence. The proof of Fatou's lemma for this example is a direct consequence of the classical case for a discrete measure plus part of the proof in Example 2.

5. Let $A=$ all $C^{1}$ functions on the circle with $C^{1}$ norm, $\mathscr{T}=$ the topology of convergence in each Fourier coefficient. If $f_{\gamma} \rightarrow f$ in $\mathscr{T}$, then $K_{n} * f_{r} \rightarrow K_{n} * f$ uniformly, since the Fejer kernel $K_{n}$ uses only finitely many coefficients. By the argument in example 2, 
$\left\|K_{n} * f\right\| \leqq \sup _{r}\left\|K_{n} * f_{r}\right\|$ and since $K_{n} * f \rightarrow f$ uniformly (and even in norm), we get

$$
\|f\| \leqq \sup _{n}\left\|K_{n} * f\right\| \leqq \sup _{n} \sup _{\gamma}\left\|K_{n} * f_{\gamma}\right\| .
$$

The proof is completed by the observation that $\left\|K_{n} * g\right\| \leqq\|g\|$ for any $g$. See [1] for abstractions and generalizations of this example.

In discussing the general situation let us use $X^{*}$ for the space of all continuous linear mappings of a topological vector space $X$ into the scalar field. Observe that $(A, \mathscr{T})^{*} \subseteq A^{*}$. If $X$ is a normed linear space, let $U(X)$ be the unit ball of $X$.

ThEorem 1. Let A, $\mathscr{T}$ be given. These are equivalent.

(1) Fatou's lemma holds for $A$ relative to $\mathscr{T}$.

(2) $(A, \mathscr{T})^{*} \cap U\left(A^{*}\right)$ is weak-*-dense in $U\left(A^{*}\right)$.

(3) For every $a \in A,\|a\|=\sup \left\{|\varphi(a)|: \varphi \in(A, \mathscr{T})^{*},\|\varphi\| \leqq 1\right\}$.

Proof. 1 $\rightarrow 3$ : It is sufficient to prove (3) for an arbitrary $a_{0}$ of norm 1. Let $C=\{a:\|a\| \leqq 1-\varepsilon\}$, where $\varepsilon>0$. By (1) the closure $\bar{C}$ of $C$ in $\mathscr{T}$ does not contain $a_{0}$. Since $\bar{C}$ is convex, circled, and contains 0 , there is a functional $\varphi \in(A, \mathscr{T})^{*}$ with

$$
\varphi\left(a_{0}\right)>\sup \{|\varphi(\alpha)|: \alpha \in \bar{C}\} \geqq \sup \{|\varphi(\alpha)|: a \in C\}=(1-\varepsilon)\|\varphi\| \text {. }
$$

Since $\left\|a_{0}\right\|=1$, this proves (3).

$3 \rightarrow 2$ : If (2) is false, let $D=(A, \mathscr{T})^{*} \cap U\left(A^{*}\right)$ and let $\Psi \in$ $U\left(A^{*}\right)$ be chosen so that $\Psi \notin \bar{D}=$ the weak-*-closure of $D$. This implies existence of $F$ in $\left(A^{*}\right.$, weak-*-topology)* with

$$
F(\Psi)>\sup \{|F(\varphi)|: \varphi \in \bar{D}\} \text {. }
$$

Since $F$ is given by evaluation at some $a \in A$, we get

$$
\Psi(a)>\sup \{|\varphi(a)|: \varphi \in \bar{D}\} \geqq \sup \{|\varphi(\alpha)|: \varphi \in D\} .
$$

This evidently contradicts (3).

$2 \rightarrow 1$ : Let $a_{\beta} \rightarrow a_{0}$ in $\mathscr{T}$. Choose $\varphi_{0} \in A^{*}$ with $\left\|\varphi_{0}\right\|=1$ and $\varphi_{0}\left(a_{0}\right)=\left\|a_{0}\right\|$. By (2) there is $\varphi_{\rho} \rightarrow \varphi_{0}($ weak $*)$ with $\left\|\varphi_{\rho}\right\| \leqq 1$ and $\varphi_{\rho} \in(A, \mathscr{T})^{*}$. Then $\left|\varphi_{\rho}\left(a_{\beta}\right)\right| \leqq\left\|\varphi_{\rho}\right\|\left\|a_{\beta}\right\| \leqq\left\|a_{\beta}\right\|$ and $\varphi_{\rho}\left(a_{\beta}\right) \underset{\beta}{\rightarrow} \varphi_{\rho}\left(a_{0}\right)$. Therefore $\left|\varphi_{\rho}\left(a_{0}\right)\right| \leqq \sup _{\beta}\left|\varphi_{\rho}\left(a_{\beta}\right)\right| \leqq \sup _{\beta}\left\|a_{\beta}\right\|$. It follows that

$$
\left\|a_{0}\right\|=\varphi_{0}\left(a_{0}\right)=\lim _{\rho} \varphi_{\rho}\left(a_{0}\right) \leqq \sup _{\beta}\left\|a_{\beta}\right\|,
$$

and this is equivalent to (1). 
Corollary. Let $z_{0}$ belong to a plane domain and let $A$ be the space of functions continuous on the closure of the domain and analytic in the interior, $\|f\|=\sup |f|$. Then every $\phi \in A^{*}$ is the weak-i-limit of functions $\Psi$ of norm $\leqq\|\varphi\|$ of the form $\Psi(f)=\sum_{\text {finite }} a_{\jmath} f^{(j)}\left(z_{0}\right)$.

Theorem 2. If $A$ is reflexive, then Fatou's lemma holds for $A$ relative to any $\mathscr{T}$.

Proof. Let $a_{r} \rightarrow a_{0}$ in $\mathscr{T}$. We need to show that $\left\|a_{0}\right\| \leqq \sup _{i}\left\|a_{i}\right\|$. If the sup is $\infty$, we are done. Let the sup be $K<\infty$. Since $A$ is reflexive, the ball of radius $K$ in $A$ is compact in the weak topology induced by $A^{*}$ and hence is compact in the topology induced by $(A, \mathscr{T})^{*} \leqq A^{*}$. This means that $a_{1}$ exists in $A$ with $\left\|a_{1}\right\| \leqq K$ and a subnet exists with $\varphi\left(a_{r(\beta)}\right) \rightarrow \varphi\left(a_{1}\right)$ for every $\varphi \in(A, \mathscr{G})^{*}$. Since $a_{r(\beta)} \rightarrow a_{0}$ in $\mathscr{T}, \varphi\left(a_{\gamma(\beta)}\right) \rightarrow{ }_{\beta} \varphi\left(a_{0}\right)$ for every $\varphi \in(A, \mathscr{T})^{*}$. This implies that $a_{0}=a_{1}$, since $\mathscr{T}$ is locally convex and Hausdorff, and we are done.

In a similar manner we obtain the following.

Theorem 3. If $A$ is the dual of a normed linear space and $\mathscr{T}$ is comparable to the weak-*-topology on A, then Fatou's lemma holds for $A$ relative to $\mathscr{I}$.

THEOREM 4. Let $A$ be any normed linear space which is not reflexive. Then there is a locally convex Hausdorff topology $\mathscr{T}$ on $A$, weaker than the norm topology, so that Fatou's lemma fails for $A$ relative to $\mathscr{T}$.

Proof. Let $\varphi \in A^{*}, \varphi \neq 0$, and put $B=\operatorname{ker} \varphi . \quad B$ is a closed subspace of $A$ and $A$ is isomorphic with $B \oplus \mathrm{C}$; hence, $B$ is not reflexive. This means that when we view $A \subseteq A^{* *}$ in the natural way, $B$ is not closed in the $A^{*}$-topology. We can choose $a_{\hat{\beta}} \in B$ and $\underline{a} \in A^{* *}-B$ so that $\left\|a_{\beta}\right\|=1$ and $a_{\beta} \rightarrow \underline{a}$ in the topology induced by $A^{*}$. Since $\varphi \equiv 0$ on $B$, it follows that $\varphi(\underline{a})=0$, and since $\underline{a} \in B$ we see that $\underline{a} \in A$.

Let $A^{\prime}$ be the span of $B$ and $\underline{a}$. For any $a_{0} \in A-B$, the mapping between $A^{\prime}$ and $A$ given by $b+\lambda \underline{a} \Leftrightarrow b+\lambda a_{0}$ is a vector space isomorphism which is bicontinuous in the norm. Use this mapping to transfer the $A^{*}$-induced topology of $A^{\prime}$ over onto $A$, where we obtain a locally convex Hausdorff topology $\mathscr{T}$ weaker than the norm topology. Since $a_{\beta} \rightarrow a_{0}$ in $\mathscr{T}$, the proof is completed by selecting $a_{0}$ to have norm 2 , for example. 
REMARK. By a slight modification of the method of proof one can obtain $\mathscr{T}$ so that each element of a sequence $a_{n},\left\|a_{n}\right\|=n$, will be the $\mathscr{T}$-limit of elements of norm $\leqq 1$. Then it will be impossible to give $A$ an equivalent norm which accommodates $\mathscr{T}$ in Fatou's lemma.

The failure of Fatou's lemma for an abstract linear space is not surprising. However, it is somewhat unexpected for $A$ a subalgebra of $C(X)$ when $\mathscr{T}$ is uniform convergence on $X$. Recall that any semi-simple commutative Banach algebra $A$ may be regarded, via the Gelfand transform, as a subalgebra of $C(\Delta), \Delta$ the maximal ideal space of $A$, and $\|f\| \geqq \sup _{\Delta}|f|$.

THEOREM 5. There exists a semi-simple commutative Banach algebra $A$ with elements $f, f_{n}$ satisfying $\left\|f_{n}\right\| \leqq 1,\left\|f_{n}\right\|>1$, and $f_{n} \rightarrow f$ uniformly on $\Delta$. That is, Fatou's lemma fails for $A$ relative to the topology of uniform convergence on its maximal ideal space.

Proof. First consider the Banach algebra $B$ of all sequences $x=$ $\left(x_{1}, x_{2}, \cdots\right)$ such that $\||x|\|\left|=\Sigma 2^{k}\right| x_{k} \mid<\infty$. All algebraic operations on $B$ are defined coordinate-wise. As a Banach space $B$ is isometrically isomorphic to $l^{1}$. We can compute the maximal ideal space of $B$ as follows. Let $h_{j}(x)=x_{j}$. These homomorphisms show that $B$ is semi-simple. To see that these are all the homomorphisms of $B$, let $h: B \rightarrow \mathrm{C}$ and look at $h\left(e_{j}\right)$, where $e_{j}=(\cdots, 0,1,0, \cdots)$ with the " 1 " in the $j^{\text {th }}$ place. If $h \neq 0$, then $h\left(e_{j_{0}}\right) \neq 0$ for some $j_{0}$, since linear combinations of the $e_{j}$ are dense. Applying $h$ to the equation $e_{i} e_{j}=\delta_{i j}$ yields $h\left(e_{j_{j}}\right)=1$ and $h\left(e_{j}\right)=0$ for $j \neq j_{0}$. Then the equation $e_{j_{0}}\left(x-x_{j_{0}} e_{j_{j}}\right)=0$ leads to $h(x)=x_{j_{0}}$ and $h \equiv h_{j_{0}}$.

Now we obtain $A$ as the algebra of $B$ together with a new norm \|\| , equivalent to \|\|$\|$. Of course, A will be semi-simple and have the same maximal ideal space. To construct the new norm, let $C=\left\{x \in B:\left|x_{1}\right| \leqq 1 / 4\right.$ and $\left.\sum_{2}^{\infty} 2^{k}\left|x_{k}\right| \leqq 1\right\}$ and let $D=$ the convex hull of $\left\{e^{i \theta}\left(e_{1} / 2+4 e_{k} / 2^{k}\right)\right.$ : all real $\theta$, all $\left.k \geqq 4\right\}$. Both $C$ and $D$ are convex and circled (stable under multiplication by scalars of absolute value $\leqq 1)$. Put $U=$ the convex hull of $C \cup D$ and $p=$ the support functional of $U: p(x)=\inf \{r: x \in r U\}=1 / \sup \{r: r x \in U\}$.

Observe that $\|\mid x\| \leqq 1 \Rightarrow 1 / 2 x \in C \Rightarrow p(x) \leqq 2$ and

$$
\begin{aligned}
p(x) \leqq 1 \Longrightarrow(1-\varepsilon) x & =\lambda c+(1-\lambda) d \Rightarrow(1-\varepsilon)|\|x \mid\| \leqq \max (\||| c|||||| d|| \mid) \\
& =\max \left(1 \frac{1}{2}, 5\right)=5
\end{aligned}
$$


Since $U$ is convex, circled, and absorbing, $p$ defines a new norm $\|x\|=p(x)$ on $B$ which we have seen is equivalent with the norm III $\| 1$.

To show that $p$ is a Banach algebra norm, it is sufficient to show $u u^{\prime} \in U$ for any $u, u^{\prime} \in U$. Since $u$ (resp. $u^{\prime}$ ) is a convex combination of $c, d$ (resp. $\left.c^{\prime}, d^{\prime}\right), u u^{\prime}$ is a convex combination of $c c^{\prime}, c d^{\prime}, c^{\prime} d$, and $d d^{\prime}$. The first three clearly belong to $C$; furthermore, $d d^{\prime} \in C$ since the definition of $D$ requires $k \geqq 4$. This proves $p$ is submultiplicative.

Now we estimate $p\left(e_{1}\right)$. Suppose $r e_{1} \in U$; then $r e_{1}=\lambda c+(1-\lambda) d$. Apply the linear functional $L(x)=\sum_{2}^{\infty} 2^{k} x_{k}$ to this equation and get $0=\lambda L(c)+(1-\lambda) L(d)$. Therefore, $\quad(1-\lambda)|L(d)| \leqq \lambda|L(c)| \leqq \lambda, \quad$ since $|L(c)| \leqq 1$. $L(d)=8 d_{1}$ for all $d$, since this is true for the generators of $D$. Hence, $(1-\lambda)\left|d_{1}\right| \leqq \lambda / 8$. Looking at the first coordinate of $r e_{1}=\lambda c+(1-\lambda) d$, we see $r=\lambda c_{1}+(1-\lambda) d_{1}$. Finally, $\left|c_{1}\right| \leqq 1 / 4$ by definition and $(1-\lambda)\left|d_{1}\right| \leqq \lambda / 8$; so $r \leqq 3 / 8 \lambda \leqq 3 / 8$. Thus $p\left(e_{1}\right) \geqq 8 / 3$.

The theorem is proved with $f=e_{1} / 2$ and $f_{n}=e_{1} / 2+4 e_{n} / 2^{n}$.

\section{REFERENCE}

1. K. de Leeuw, Linear spaces with a compact group of operators, Illinois. J. Math., 2 (1958), 367-377.

Received February 18, 1971. This research was supported in part by NSF Grant 11911. 


\section{PACIFIC JOURNAL OF MATHEMATICS}

\section{EDITORS}

H. SAMELSON

Stanford University

Stanford, California 94305

C. R. Hовву

University of Washington

Seattle, Washington 98105
J. DUGUNDJI

Department of Mathematics

University of Southern California

Los Angeles, California 90007

RICHARD ARENS

University of California

Los Angeles, California 90024

\section{ASSOCIATE EDITORS}
E. F. BECKENBACH
B. H. NeumanN
F. WOLF
K. YoSHIDA

\section{SUPPORTING INSTITUTIONS}

UNIVERSITY OF BRITISH COLUMBIA

CALIFORNIA INSTITUTE OF TECHNOLOGY

UNIVERSITY OF CALIFORNIA

MONTANA STATE UNIVERSITY

UNIVERSITY OF NEVADA

NEW MEXICO STATE UNIVERSITY

OREGON STATE UNIVERSITY

UNIVERSITY OF OREGON

OSAKA UNIVERSITY

UNIVERSITY OF SOUTHERN CALIFORNIA
STANFORD UNIVERSITY

UNIVERSITY OF TOKYO

UNIVERSITY OF UTAH

WASHINGTON STATE UNIVERSITY

UNIVERSITY OF WASHINGTON

AMERICAN MATHEMATICAL SOCIETY CHEVRON RESEARCH CORPORATION NAVAL WEAPONS CENTER 


\section{Pacific Journal of Mathematics}

\section{Vol. 38, No. $1 \quad$ March, 1971}

Bruce Alan Barnes, Banach algebras which are ideals in a Banach algebra ..... 1

David W. Boyd, Inequalities for positive integral operators............... 9

Lawrence Gerald Brown, Note on the open mapping theorem .............. 25

Stephen Daniel Comer, Representations by algebras of sections over Boolean

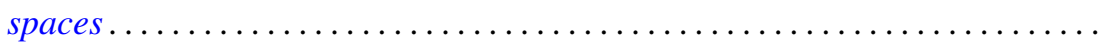

John R. Edwards and Stanley G. Wayment, On the nonequivalence of

conservative Hausdorff methods and Hausdorff moment sequences ........

P. D. T. A. Elliott, On the limiting distribution of additive functions $(\bmod 1) \ldots \ldots$

Mary Rodriguez Embry, Classifying special operators by means of subsets

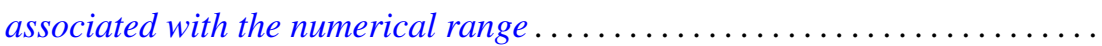

Darald Joe Hartfiel, Counterexamples to a conjecture of G. N. de Oliveira ......

C. Ward Henson, A family of countable homogeneous graphs...............

Satoru Igari and Shigehiko Kuratsubo, A sufficient condition for

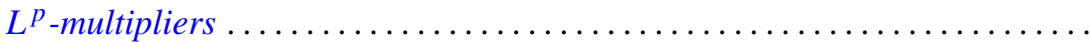

William A. Kirk, Fixed point theorems for nonlinear nonexpansive and

generalized contraction mappings............................

Erwin Kleinfeld, A generalization of commutative and associative rings ...... 95

D. B. Lahiri, Some restricted partition functions. Congruences modulo $11 \ldots \ldots 103$

T. Y. Lin, Homological algebra of stable homotopy ring $\pi *$ of spheres ....... 117

Morris Marden, A representation for the logarithmic derivative of a meromorphic function...........................

John Charles Nichols and James C. Smith, Examples concerning sum properties for metric-dependent dimension functions . .

Asit Baran Raha, On completely Hausdorff-completion of a completely

Hausdorff space.

M. Rajagopalan and Bertram Manuel Schreiber, Ergodic automorphisms and affine transformations of locally compact groups..........

N. V. Rao and Ashoke Kumar Roy, Linear isometries of some function

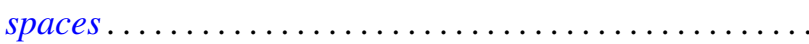

William Francis Reynolds, Blocks and F-class algebras of finite groups

Richard Rochberg, Which linear maps of the disk algebra are multiplicative ...

Gary Sampson, Sharp estimates of convolution transforms in terms of decreasing

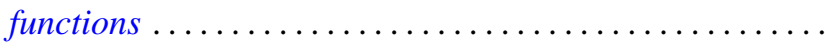

Stephen Scheinberg, Fatou's lemma in normed linear spaces

Ken Shaw, Whittaker constants for entire functions of several complex

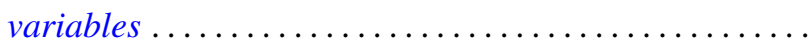

James DeWitt Stein, Two uniform boundedness theorems................ 251

$\mathrm{Li} \mathrm{Pi} \mathrm{Su,} \mathrm{Homomorphisms} \mathrm{of} \mathrm{near-rings} \mathrm{of} \mathrm{continuous} \mathrm{functions} \mathrm{.} \mathrm{.............} 261$

Stephen Willard, Functionally compact spaces, $C$-compact spaces and mappings of minimal Hausdorff spaces....................... 